\title{
Metagenomic analysis and the functional profiles of traditional fermented pork fat 'sa-um' of Northeast India
}

\author{
Surajit De Mandal', Sambanduram Samarjit Singh', Rajendra Bose Muthukumaran², Kawl Thanzami , \\ Vinod Kumar ${ }^{4}$ and Nachimuthu Senthil Kumar ${ }^{1 *}$ (D)
}

\begin{abstract}
Fermented pork fat (sa-um) is traditionally and extensively consumed in Northeast Indian region for several decades. However, no scientific reports are available regarding its nutritional value as well as its potential health risks. The objective of this work was essentially the characterization of sa-um using a polyphasic approach, viz., physicochemical, electrospray ionization-mass spectrometry (ESI+ ${ }^{+} \mathrm{MS}$ ) and metagenomic analysis in order to gain an understanding of the nutrient contents and microbial population diversity. On a dry weight basis, about $91 \%$ fat, $2 \%$ carbohydrate and $0.70 \%$ protein were present. ESI ${ }^{+}-\mathrm{MS}$ analysis of sa-um revealed the presence of various polar and neutral lipids corresponding to monoacylglyceride, diacylglyceride and triacylglyceride species. The dominant bacterial phyla were Firmicutes, Proteobacteria and Bacteroidetes. A total of 72 bacterial genera were identified, largely abundant with Clostridium species including C. butyricum, C. citroniae, C. methylpentosum, C. perfringens, C. saccharogumia and C. tetani. The imputed functional profiles of bacterial communities were predominantly involved in energy, carbohydrate and amino acid metabolisms. Furthermore, this study deduces the presence of pro-inflammatory molecules as well as antibiotic resistance genes associated with the bacterial families such as Bacillaceae, Bacteroidaceae, Clostridiaceae, Corynebacteriaceae and Enterobacteriaceae which might be a major health concern for the sa-um consuming population.
\end{abstract}

Keywords: Pork fat, Sa-um, Nutrient content, Bacterial community, Food safety

\section{Introduction}

Fermentation is regarded as an ancient and economical method for value addition and preservation of foodstuff in Northeast India (Tamang 1998). The indigenous foods are used from ancient times and directly related with the tradition and culture (Sekar and Mariappan 2007). However, the preparation of indigenous or traditional fermented foods only remains as a household art today (Beuchat 2008). The nutritional value of the food is concomitantly augmented with the increase in vitamin content and protein solubility during fermentation (Sohliya et al. 2009). Fermentation also serves as a potential source

\footnotetext{
*Correspondence: nskmzu@gmail.com

${ }^{1}$ Department of Biotechnology, Mizoram University, Aizawl, Mizoram 796004, India

Full list of author information is available at the end of the article
}

of bioactive compounds which may provide antimicrobial, cholesterol-lowering ability as well as antithrombotic and antioxidative activities (Hartmann and Meisel 2007). Diverse ethnic communities of Northeast India prepare various kinds of fermented food products and use them as a basic component of their diet (Tamang et al. 2009). Fermented pork fat is being consumed by people from different parts of the world as a source of daily food (Aquilanti et al. 2007). Sa-um, an indigenous animal fat product is semi-dry, gummy, 'ripened' lard made with caul fat adipose tissue and it has no appreciable organoleptic qualities although it exhibits distinct astringency. It presents negative health attributes due to high saturated fat/cholesterol content as it is derived from pork fat (Hooper et al. 2001). Sa-um preparation takes place on a cottage-industrial scale in households 
which does not have firmly established procedures and as a result the production process fluctuates on a seasonal basis. Sa-um, has peculiar sensorial attributes (smell and taste) due to ripening process besides the enzymatic lipolytic activities of the microbial populations present in it. Pork and beef products are often associated with different microbial populations (Borch et al. 1996; Nieminen et al. 2011; Pennacchia et al. 2011). However, no detailed study has been carried out on the chemical or microbiological characteristics of sa-um.

Exploration of bacterial community using conventional methods provides limited information than the actual diversity. In recent years, next generation sequencing (NGS) technology has considerably enhanced our ability to assess and understand the microbial communities, and it provides a better understanding of the complex interactions among the diverse bacterial species in a specific community. NGS has been employed to investigate the microbial communities of various foods (Solieri et al. 2013; Mayo et al. 2014).

Mass spectrometry methods are rapid, robust, and reliable tool for the lipid profiling in plant and animal derived fat products (Goodacre et al. 2002; Kurata et al. 2005). In this study, we report the taxonomic composition of microbial community and their putative metabolic functions in sa-um and also compared with the other published fermented pork metagenomes (Połka et al. 2014). This is the first attempt to evaluate the nutrient content as well as bacterial diversity of $s a-u m$, and to ascertain the nutritional value and safety attributes with respect to human health.

\section{Materials and methods}

\section{Preparation method of sa-um and sample collection}

Caul fat adipose tissue is normally obtained from butcher's yard, within $6 \mathrm{~h}$ of slaughtering the animal, boiled with minimum amount of water for about 15 min (rendering) and allowed to cool. When caul fat is not available, sub-cutaneous fat adipose tissue is also employed for the production process. Boiled caul fat adipose tissue is transferred to a dry bottle gourd container for 'ripening' of the adipose tissue. The container is kept for 3-5 days under the sun for ripening during dry winter season, while it may be kept near cooking stove $(\sim 10-15 \mathrm{~cm}$ from cooking stove) for 2-3 days during rainy monsoon season. After the ripening and dehydration process, consequently the caul fat loses most of its natural and added water content and it becomes semi-dry with gummy, soft, and spongy texture. Traditional method of production of sa-um is given in Figs. 1, 2. Six sa-um samples $(200 \mathrm{~g})$ were collected from different parts of Mizoram, Northeast India, and mixed thoroughly in a sterile $500 \mathrm{ml}$ polypropylene container. Collected samples were brought immediately to the laboratory in cold box, stored at $4{ }^{\circ} \mathrm{C}$ and subsequently used for analysis within 1-2 days of collection.

\section{Biochemical analysis}

Moisture, ash, crude fiber, protein, fat, carbohydrates, calorific value, iron, zinc, sodium, calcium, magnesium, potassium content of $s a-u m$ were determined according to AOAC methods (AOAC 1995). The $\mathrm{pH}$ of samples was determined using a $\mathrm{pH}$ meter (Eutech, India).

\section{Mass spectrometry analysis}

$2.5 \mathrm{~g}$ of $s a-u m$ samples were kept in dichloromethane: methanol or acetonitrile: methanol as solvent systems for overnight followed by sonication for $10 \mathrm{~min}$. The samples were vortexed for another $5 \mathrm{~min}$ and centrifuged. The organic solution layer was subjected to mass spectrometry analysis. ESI-tandem mass spectrometry measurements were carried out with a Waters QTOF-Micromass spectrometer with nitrogen as carrier gas (flow rate $100 \mathrm{l} /$ hr) and the sample flow rate was $0.2 \mathrm{ml} / \mathrm{min}$ with the desolvation temperature of $150{ }^{\circ} \mathrm{C}$. Mass Spectra were recorded by electrospray ionization and the source voltage was maintained at $2.3 \mathrm{kV}$ in the positive ion mode.

HP-TLC analysis was performed to analyze the lipid component present in sa-um.

\section{DNA isolation, PCR and sequencing}

Isolation of the metagenomic DNA from sa-um samples were carried out using the Fast DNA spin kit (MP Biomedical, USA) and quantified by microplate reader (Spectra Max 2E, Molecular Devices, USA). The V4 hyper variable region of the $16 \mathrm{~S}$ rRNA gene was amplified by $10 \mathrm{pmol} / \mu \mathrm{l}$ of each forward 515F $\left(5^{\prime}-3^{\prime}\right)$ and reverse 806R $\left(5^{\prime}-3^{\prime}\right)$ primers. The amplification mix contained $40 \mathrm{mM}$ dNTPs (NEB, USA); $5 \times$ Phusion HF buffers (NEB, USA); $2 \mathrm{U} / \mu \mathrm{l}$ F-540 Special Phusion HS DNA Polymerase (NEB, USA); and 5 ng DNA and Milli-Q water to make up $30 \mu \mathrm{l}$ total volume. PCR conditions consisted of initial denaturation at $98{ }^{\circ} \mathrm{C}$ for $30 \mathrm{~s}$ followed by 30 cycles of $98^{\circ} \mathrm{C}$ for $10 \mathrm{~s}, 72{ }^{\circ} \mathrm{C}$ for $30 \mathrm{~s}$, and a final extension at $72{ }^{\circ} \mathrm{C}$ for $5 \mathrm{~s}$. Paired end Illumina MiSeq sequencing $(2 \times 250 \mathrm{bp})$ was performed at SciGenom Lab, Cochin, India (De Mandal et al. 2016, 2017).

\section{Sequencing analyses}

Raw fastq sequences were processed and analyzed using the QIIME software package v.1.8.0. (Caporaso et al. $2009,2010)$. Sequences with a quality score $<25$ and read length $<200$ bp were filtered and chimeric sequences were remove using USEARCH (Edgar et al. 2011). 
Raw material (Raw fresh pig fats from market)

$\downarrow$

Cleaned and washed

$\downarrow$

Boil/Cooked

$\downarrow$

Chopped into pieces

$\downarrow$

Placed in a special container called 'sa-um bur' (made from Lagenaria siceraria)

$\downarrow$

The container is placed over the fireplace for fermentation

$\downarrow$

Incubate for three days

$\downarrow$

Fermented

$\downarrow$

Sa-um

Fig. 1 Traditional method of sa-um preparation

Preprocessed V4 sequences were clustered into operational taxonomic units (OTU's) using the Uclust program (similarity cutoff $=0.97) \quad($ Edgar 2010). Representative sequence for each OTU was classified using Greengenes database (De Mandal et al. 2016, 2017; DeSantis et al. 2006).

\section{Imputed functional analysis}

The imputed functional profile of the bacterial community was performed using PICRUSt (Langille et al. 2013). In brief closed-reference, OTU was generated at a $97 \%$ similarity level and normalized using "normalize_by_copy_number.py” script. Functional profiles were predicted against KEGG database. Contributions of various taxa to different KOs were computed with the script metagenome_contributions.py (Langille et al. 2013).

\section{Comparison of sa-um with Italian salami}

Metagenome data was retrieved from SRA (Sequence Read Archives) NCBI in fastq format to compare present studies with the previously published fermented pork (Italian salami) (Połka et al. 2014). Only the samples with no starter culture (SRR5298557, SRR5298565, SRR5298588, SRR5298586) were used in our study and the good quality sequences were compiled into a single fasta file and processed using QIIME pipeline. The compositional similarity between the fermented pork 


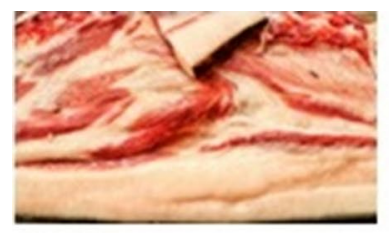

a

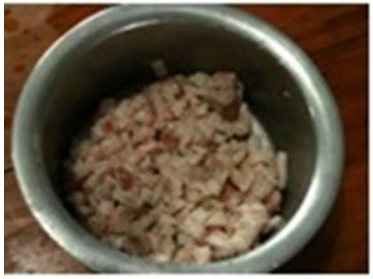

d

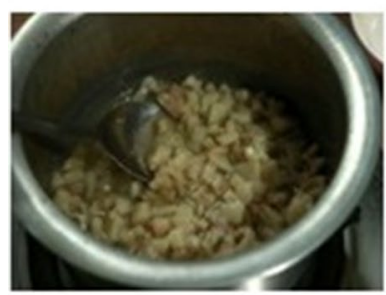

g

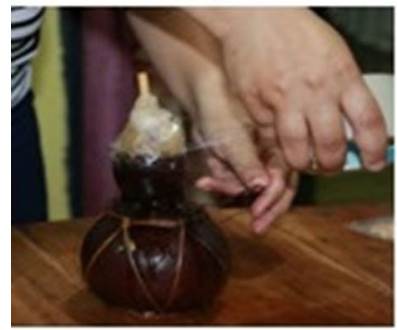

j

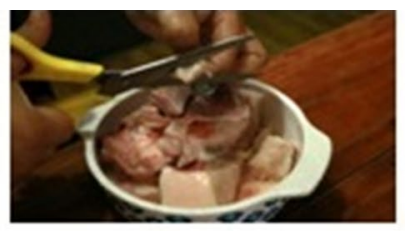

b

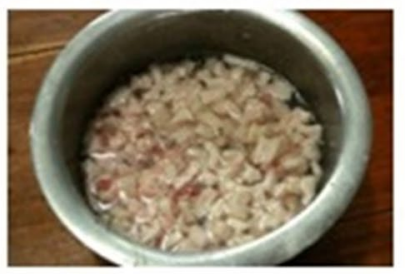

e

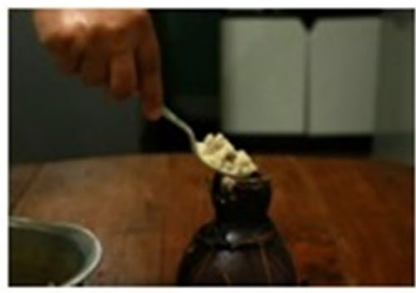

h

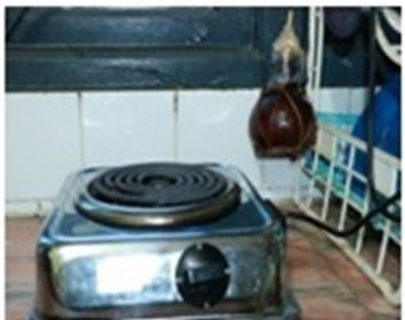

$\mathbf{k}$

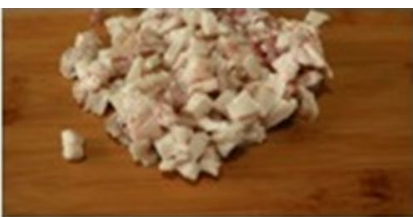

C

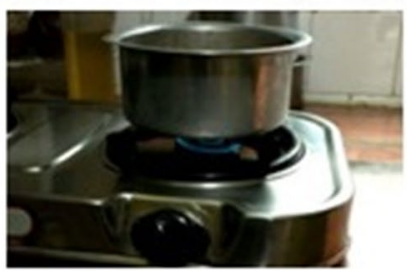

f

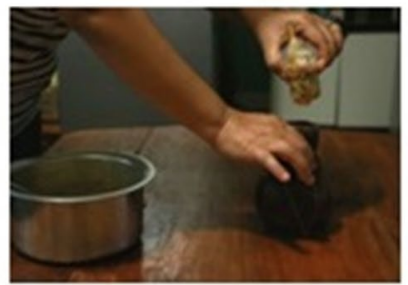

i

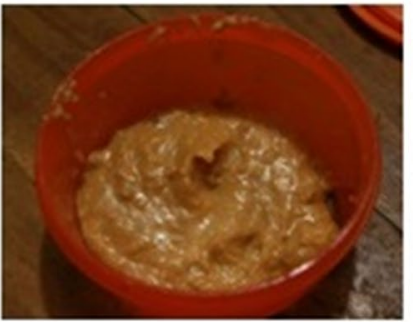

I

Fig. 2 Different steps involved in sa-um preparation. a Pork lard, $\mathbf{b}-\mathbf{d}$ mincing of lard, $\mathbf{e}-\mathbf{g}$ added water and cooked till water evaporates out, $\mathbf{h}-\mathbf{j}$ cooked lard transferred to cleansed and dried 'Um', $\mathbf{k}$ incubated at fireplace or sun, I finished product sa-um

metagenome was compared using Bray-Curtis measure for estimation of beta diversity (Bray and Curtis 1957).

\section{Results}

\section{Biochemical and mass spectrometry analysis of sa-um}

As a major constituent, the fat content accounted for 91\% followed by moisture content (6.21\%) besides trace amounts of carbohydrate, crude fiber and ash. The high fat content of sa-um is reflected in its calorific value $(830 \mathrm{kcal} / 100 \mathrm{~g})$ and the $\mathrm{pH}$ was slightly acidic (6.60). Physicochemical compositions of the fermented pork fat sa-um are summarized in Table 1. Preliminary
$\mathrm{ESI}^{+}$-MS analysis of $s a-u m$ has indicated the presence of various polar and neutral lipid molecular species in the range from 295 to 920 Dalton and these lipid components are essentially clustered in the mass range of 300, 600, 900 that corresponds to monoacylglyceride, diacyl-glyceride, and triacylglyceride molecular species with a noticeable presence of the myristic acid (C14:0) in acylglycerides besides sterols (Fig. 3). The observed glycerides consist of acyl sidechains corresponding to fourteen carbons or longer, while the neutral lipid molecular components comprise unsaturated fatty acid glycerides (mono and poly) as well as saturated 
Table 1 Biochemical analysis of sa-um

\begin{tabular}{ll}
\hline & Value \\
\hline Major nutrients (\% by wt.) & \\
Moisture & 6.21 \\
Total ash & 0.1 \\
Fat & 91 \\
Crude fiber & BDL of 0.1 \\
Protein & 0.7 \\
Carbohydrates & 2 \\
Calorific value (Kcals/100 g) & 830 \\
Minerals (mg/100 g) & \\
Iron & 0.2 \\
Zinc & 0.07 \\
Sodium & 4.73 \\
Calcium & 2.61 \\
Magnesium & 0.45 \\
Potassium & 3.96 \\
\hline BDLbelow detection & \\
\hline
\end{tabular}

$B D L$ below detection limit

fatty acid containing acylglycerides. HP-TLC analysis showed the presence of lipid components with a wide range of polarity that commensurate with the mass spectrometric analysis of sa-um (Additional file 1: Figure S1).

\section{Phylogenetic analysis of the bacterial community}

A total of 263,741 paired-end raw reads were obtained from the sa-um metagenome. After pre-processing, 60,117 reads were obtained and clustered into 1348 OTU's with $97 \%$ sequence similarity. Further analyses detected 627 singletons and were subsequently removed from the OTU table. Finally, 722 OTU's were taken for downstream analysis (Table 2). Dominant phyla were Firmicutes (52.91\%), Proteobacteria (10.8\%), Bacteroidetes (9.6\%), Actinobacteria (4.98\%), Chloroflexi (2.21\%), Planctomycetes (2.07\%), Synergistetes (1.193\%) and Acidobacteria (1.1\%). Other minor identified phyla $(<1 \%)$ were Cyanobacteria, Verrucomicrobia, AD3, Thermi, Armatimonadetes, Tenericutes, Crenarchaeota, GAL15, Gemmatimonadetes, Euryarchaeota, Nitrospirae and Chlorobi. However, more than $10 \%$ of the total OTU's remained unidentified. The present study identified 722 OTU's belonging to three major groups of bacteria: Firmicutes, Proteobacteria and Bacteroidetes (Fig. 4).

Firmicutes essentially occupied more than half of total OTU's present in the complete dataset. 82 OTU's (52.91\%) consisted of 39,046 (65.63\%) reads assigned under the phylum Firmicutes, of which three classes (Bacilli, Clostridia and Erysipelotrichia), six orders (Clostridiales, Bacillales, OPB54, Lactobacillales, Erysipelotrichales and Thermoanaerobacterales), and ten families (Christensenellaceae, Clostridiaceae, Enterococcaceae, Erysipelotrichaceae, Lachnospiraceae, Planococcaceae, Ruminococcaceae, Staphylococcaceae, Streptococcaceae and Veillonellaceae) were identified. Top four dominant OTU's under this genus were Clostridium tetani (21.29\%), B. coagulans (0.47\%), Phascolarctobacterium sp. (0.32\%) and C. perfringens (0.31\%). Other identified genera includes Anaerotruncus, Blautia, Coprobacillus, Coprococcus, Dialister, Dorea, Enterococcus, Faecalibacterium, Lactobacillus, Marinococcus, Megamonas, Megasphaera, Oscillospira, Phascolarctobacterium, Roseburia, Ruminococcus, Ruminococcus, SMB53, Staphylococcus, Streptococcus and Veillonella.

Proteobacteria represented the second dominant phyla. A total of 78 (10.8\%) OTU's and 14,371 (24.16\%) reads were affiliated with the phylum Proteobacteria. Five classes (Alphaproteobacteria, Betaproteobacteria, Deltaproteobacteria, Epsilonproteobacteria and Gammaproteobacteria) and thirteen orders (Aeromonadales, Alteromonadales, Burkholderiales, Campylobacterales, Desulfobacterales, Enterobacteriales, Enterobacteriales, Oceanospirillales, Pseudomonadales, Rhizobiales, Rhodobacterales, Vibrionales and Xanthomonadales) were indentified under this phylum. Dominant OTU's under this phylum were classified under the genera Acinetobacter, Citrobacter, Escherichia, Halomonas, Proteus and Ralstonia.

The phylum Bacteroidetes consisted of two classes: Bacteroidia and Flavobacteriia. Identified families were Barnesiellaceae, Odoribacteraceae, Weeksellaceae, Bacteroidaceae, Porphyromonadaceae, Prevotellaceae, Rikenellaceae and S24-7. Identified genera under this phylum were AF12, Barnesiella, Bacteroides, Butyricimonas, Parabacteroides, Odoribacter, Prevotella and Wautersiella. 36 OTU's comprising 4116 reads belonged to Actinobacteria. Identified genera were Actinomycetospora, Adlercreutzia, Bifidobacterium, Brevibacterium, Collinsella, Corynebacterium, Kocuria, Micrococcus and Slackia. The most dominant OTU under this phylum was OTU1271 (6.4\%) was classified as C. variabile.

Two OTU's were found under the phylum Chloroflexi which belonged to FFCH10602. Five OTU's with 34 reads were classified under Verrucomicrobia. Identified genera were DA101 and Akkermansia muciniphila. Eight OTU's comprising 34 reads were classified under the phylum Acidobacteria. Identified orders include iii1-15, RB41, Ellin6513, RB41, Nov-24, RB41, Acidobacteriales and Ellin6513. OTU834 was classified under the genus Methanobrevibacter only eukaryobacteria found in this study. Only two OTU's were classified under Deinococcus (phylum Thermi). Three OTU's classified under the phylum AD3. Identified classes were ABS-6 and JG37-AG-4. Two phylotype (OTU 294 and OTU 916) were classified under 


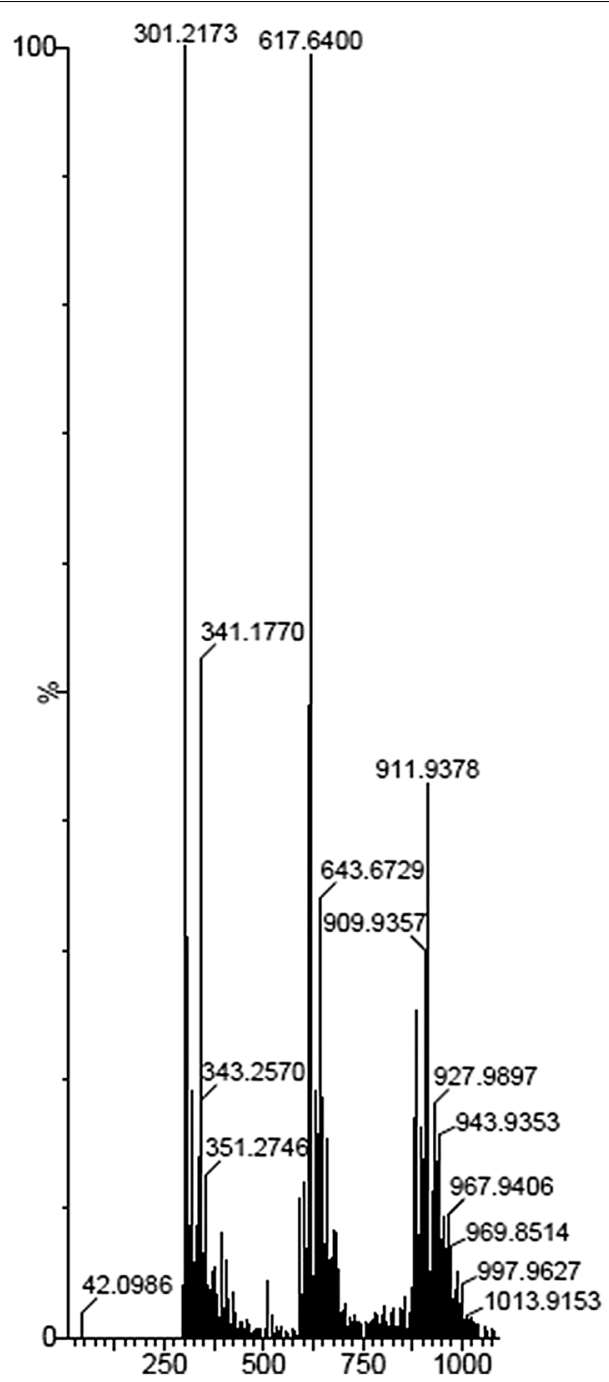

Fig. 3 Mass spectral trace of sa-um by $\mathrm{ESI}^{+}{ }^{+} \mathrm{MS}$ method. $\mathrm{m} / \mathrm{z}$ : 300-351-monoacyl glycerol, with higher intensity around m/z: 300304 region due to arachidonic acid, typical fatty acid of non-ruminant species; m/z: 550-650-diacyl glycerol species; m/z: 750-850-triacyl glycerol species; with a modest levels of triacyl phospholipid species in the $\mathrm{m} / \mathrm{z}$ : 900 region, due to smaller quantity of muscle adipose tissues in sa-um
Armatimonadetes. A total of 74 OTU's identified at the genus level were dominated by Clostridium (7.61\%), Bacteroides (4.57\%), Oscillospira (4.15\%), Corynebacterium (1.80\%), Megamonas (1.52\%), Faecalibacterium (1.38\%), Proteus (1.38\%), Ruminococcus (1.24\%), Prevotella $(1.10 \%)$ and unknown genus (58.31\%). Phylogenetic tree based on the taxonomically identified genera is shown in Fig. 5.
Imputed functional analysis

Based on the predicted metagenomes, major gene families (45\%) belonged to metabolic pathways involved in amino acid, carbohydrate, energy, nucleotide, cofactors and vitamins, lipid and xenobiotics biodegradation metabolism, enzyme families, glycan biosynthesis and metabolism, metabolism of terpenoids and polyketides, and biosynthesis of other secondary metabolites (Fig. 6). The dominant bacterial genera contributing to the antibiotic resistance were Acinetobacter, Bacillus, Bacteroides, Bifidobacterium, Blautia, Clostridium, Corynebacterium, Erwinia, Flexispira, Halomonas, Klebsiella, Megamonas, Megasphaera, Parabacteroides, Proteus, Pseudomonas, Ruminococcus, Sporolactobacillus and Wautersiella. Genes encoding for flagellin was associated with the genera Bacillus, Clostridium, Erwinia, Flexispira, Halomonas, Lysinibacillus, Megamonas, Oscillospira, Proteus, Pseudomonas, Ruminococcus, SMB53, Solibacillus and Sporolactobacillus. Genes encoding for Lipid A synthesis were deduced to occur primarily in Acinetobacter, Bacteroides, Bifidobacterium, Corynebacterium, Dialister, Erwinia, Flexispira, Halomonas, Klebsiella, Megamonas, Megasphaera, Parabacteroides, Prevotella, Proteus, Pseudomonas and Wautersiella (Table 3).

\section{Comparison of sa-um with Italian salami}

Four fermented pork (Italian Salami) meta-genome samples were compared with sa-um microbiota in order to ascertain the bacterial community of fermented products. The resultant analysis revealed that these $s a-u m$ microbiota populations are quite different in terms of composition and the relative proportion of bacterial communities. It was found that both Italian salami and sa-um were dominated with phylum Firmicutes and Proteobacteria. However, the Italian salami was dominated by the class Bacilli, whereas sa-um was enriched with Clostridia. The beta diversity analysis using unweighted Unifac approach also revealed that the $s a-u m$ contained unique bacterial communities in comparison with the Italian salami samples (Fig. 7).

\section{Discussion}

Microbial food safety and preservation are the important criteria for the consumers and allied industries. The food borne illnesses and hazardous intoxications by undesired microbial populations is still a matter of concern (EFSA and ECDC, 2013). The purpose of the study was to analyze the chemical composition and the bacterial diversity of fermented pork fat sa-um. Illumina sequencing was used to capture the complete bacterial diversity in order to assess the microbial safety of sa-um.

Higher fat content in $s a-u m$ is due to exclusive use of pork caul fat as the raw material. The observation of 
Table 2 Raw read summary of sa-um using Illumina sequencing

\begin{tabular}{ll}
\hline Total reads (paired-end) & 263,741 \\
Sequence length (bp) & 250 \\
Total data (Mb) & 131.87 \\
\%GC & 52.74 \\
Average base quality (phred score) & 35.11 \\
Passed conserved region filter & 172,188 \\
Passed spacer & 171,871 \\
Passed read quality filter & 170,843 \\
Passed mismatch filter & 60,117 \\
Consensus reads & 60,117 \\
Chimeric sequences & 0 \\
Pre-processed reads & 60,117 \\
Total OTUs picked & 1348 \\
Total singleton OTUs & 627 \\
Total OTUs after singleton removal & 722 \\
\hline
\end{tabular}

monoacyl and diacylglycerides in addition to triacylglycerides in sa-um is indicative of the enzymatic lipolytic activity by the microbial populations. It is probable that the enzymatic lipolytic activity of microbial populations could have rendered the peculiar flavour and characteristics of sa-um. Although the free fatty acids were not detected in this preliminary study, presence of free fatty acids is not ruled out as the lipids were extracted from sa-um using dicholoromethane:methanol or acetonitrile:methanol as solvent systems (Schieber 2008; Lorenzo and Franco 2012). The observed class composition of lipids in sa-um with high levels of sterols, triacylglycerols (with high levels of saturated fatty acids) and phospholipids is the characteristics of lard (Indrasti et al. 2010).
Although fermented food is a regular ingredient in the diet of many communities around the world, yet their indigenous production remains crude and rudimentary. Fermentation under largely unhygienic conditions results in the reduction in the beneficial attributes of fermentation and often leads to the growth of harmful pathogens, thereby constituting microbiological hazards. However, no inoculation of starter culture is being used for sa-um production. The anaerobic fermentation inside the closed vessel might play a role in eliminating the growth of aerobic spoilage bacteria. In the present study, majority of the classified reads belonged to the phylum Firmicutes. A total of 72 bacterial genera were identified, indicating the presence of complex and abundant bacterial communities. The metabolic by-products of these abundant bacterial members plays a key role in the formation of its unique flavours (Huang et al. 2017).

To ensure the safety and appeal of indigenous food products, it is necessary to determine presence of pathogens in them. Highest numbers of OTU falls under the genus Clostridium. This may be due to fact that the higher fat content, as a result of the lipolytic processes, may be creating an anaerobic environment and thereby inhibiting most of the aerobic microorganisms. Six Clostridium species was identified in sa-um were $C$. butyricum, $C$. citroniae, C. methylpentosum, C. perfringens, C. saccharogumia and C. tetani. In general, C. butyricum is used as a probiotics agent and employed to treat antimicrobial and non-antimicrobial associated diarrhea, constipation, and irritable bowel syndrome (Seki et al. 2003; Shimbo et al. 2005). However, both C. perfringens is a major concern in meat or fermented meat products (Akhtar et al. 2009; Golden et al. 2009; Linton et al. 2014).

The present study also identified the genus Helicobacter in sa-um. Some members under this genus are
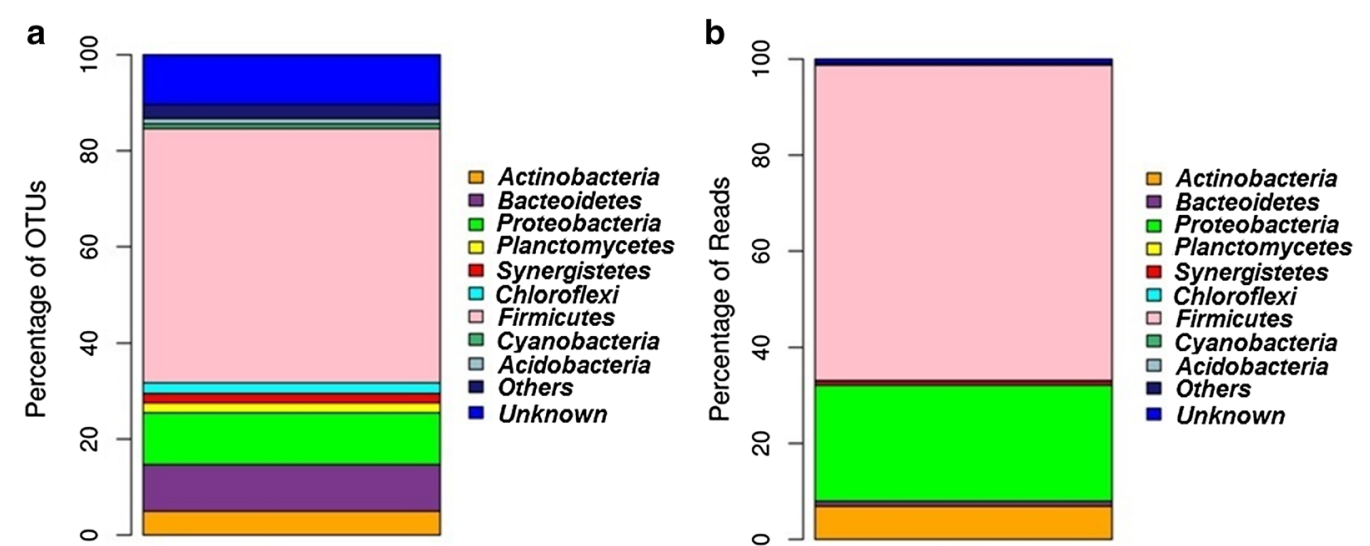

Fig. 4 Taxonomy classification of OTU's at phylum level (a) and reads at phylum level (b) from sa-um. Only top 10 enriched phyla categories are shown in the figure 


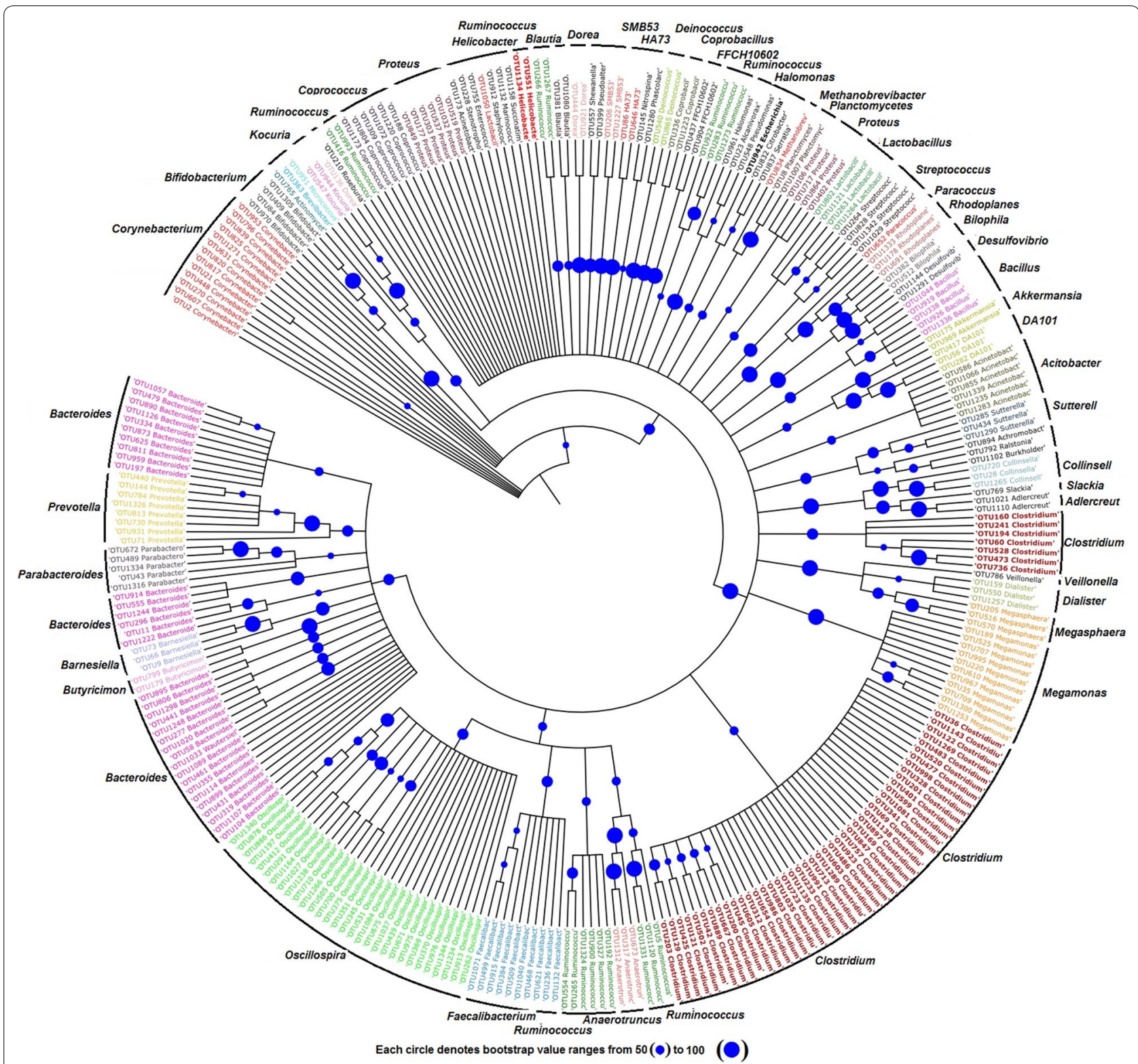

Fig. 5 Phylogenetic tree based on the identified genera using neighbour joining method

pathogenic and found to cause peptic ulcers, gastritis, and stomach cancer (Yamaoka 2008). This is a significant risk factor for high incidence of stomach cancer among the populace of Mizoram (Ghatak et al. 2016). Identified bacterial genera possibly involved in fermentation of sa-um were Kouria, Lactobacillus, B. coagulans, Collinsella, and Coprococcus. The genus Kouria is a usually considered as non-pathogenic bacteria which are rarely associated with human infections (Nam et al. 2012). B. coagulans is a lactic acid-forming species having the property of both genera Lactobacillus and Bacillus and is often used as probiotics in pigs, cattles and shrimp (Hammer 1915; Sanders et al. 2003). A member under the genus Collinsella was capable of fermenting carbohydrates and thus produces hydrogen gas and ethanol (Goldman 2015). The anaerobic cocci, Coprococcus actively ferments carbohydrates and produces butyric, acetic, formic and/or lactic acids. Veillonella are Gram-negative anaerobic cocci, well known for its lactate fermenting abilities. However, the observed mild acidic $\mathrm{pH}$ of $s a-u m(\mathrm{pH}=6.60)$ is indicative of the production of organic acids at modest levels due to the fermentation of negligible carbohydrate content in raw material and/or sa-um. Recently, the informative software PICRUSt was used to study the functional potential of the bacterial community using $16 \mathrm{~S}$ rRNA 


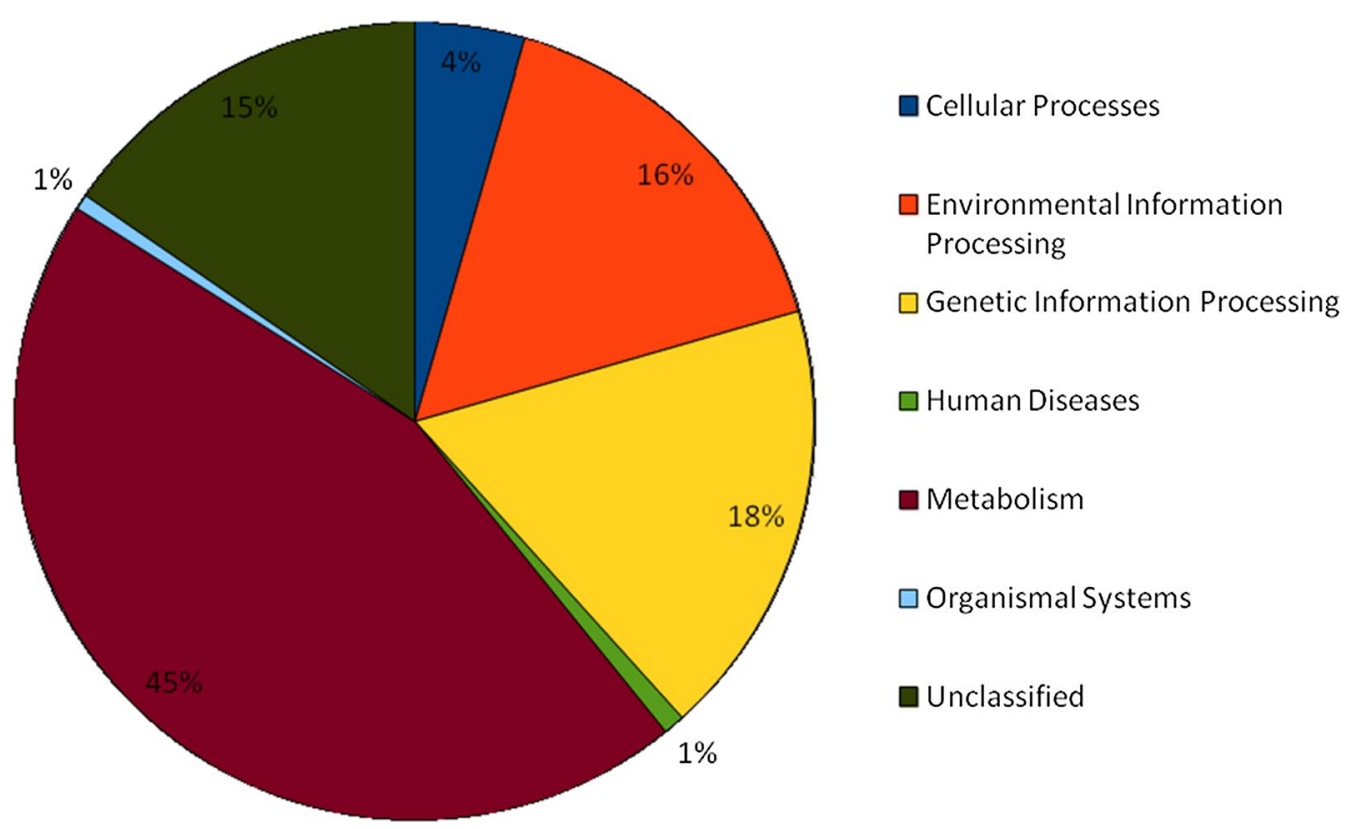

Fig. 6 Distribution of KEGG pathway in sa-um metagenome

Table 3 PICRUSt predicted genes and corresponding bacterial genus involved in antibiotic resistance and proinflammatory activities in sa-um

\begin{tabular}{|c|c|c|c|c|c|c|c|c|c|}
\hline \multicolumn{5}{|c|}{ Pro-inflammatory genes } & \multicolumn{5}{|c|}{ Antibiotic resistance genes } \\
\hline K00748 & K02406 & K02517 & K11693 & K11694 & K05595 & K07552 & K08170 & K07694 & K08221 \\
\hline Acinetobacter & Bacillus & Acinetobacter & $\begin{array}{l}\text { Candidatus } \\
\text { Arthromitus }\end{array}$ & $\begin{array}{l}\text { Staphylococ- } \\
\text { cus }\end{array}$ & Bacteroides & Acinetobacter & Clostridium & $\begin{array}{l}\text { Ruminococ- } \\
\text { Cus }\end{array}$ & Bacillus \\
\hline Akkermansia & Clostridium & Bacteroides & $\begin{array}{l}\text { Staphylococ- } \\
\text { cus }\end{array}$ & & $\begin{array}{l}\text { Bifidobacte- } \\
\text { rium }\end{array}$ & Bacteroides & Klebsiella & Blautia & $\begin{array}{l}\text { Sporolacto- } \\
\text { bacillus }\end{array}$ \\
\hline Bacteroides & Erwinia & $\begin{array}{l}\text { Bifidobacte- } \\
\text { rium }\end{array}$ & $\begin{array}{l}\text { Exiguobacte- } \\
\text { rium }\end{array}$ & & Erwinia & Clostridium & & & \\
\hline Dialister & Flexispira & $\begin{array}{l}\text { Corynebacte- } \\
\text { rium }\end{array}$ & & & Klebsiella & $\begin{array}{l}\text { Corynebacte- } \\
\text { rium }\end{array}$ & & & \\
\hline Erwinia & Halomonas & Dialister & & & $\begin{array}{l}\text { Parabacte- } \\
\text { roides }\end{array}$ & Erwinia & & & \\
\hline Flexispira & Lysinibacillus & Erwinia & & & Proteus & Flexispira & & & \\
\hline Halomonas & Megamonas & Flexispira & & & Pseudomonas & Halomonas & & & \\
\hline Klebsiella & Oscillospira & Halomonas & & & Wautersiella & Klebsiella & & & \\
\hline Megamonas & Proteus & Klebsiella & & & & Megamonas & & & \\
\hline Megasphaera & Pseudomonas & Megamonas & & & & Megasphaera & & & \\
\hline $\begin{array}{l}\text { Parabacte- } \\
\text { roides }\end{array}$ & Ruminococcus & Megasphaera & & & & Proteus & & & \\
\hline Prevotella & SMB53 & $\begin{array}{l}\text { Parabacte- } \\
\text { roides }\end{array}$ & & & & Pseudomonas & & & \\
\hline Proteus & Solibacillus & Prevotella & & & & Wautersiella & & & \\
\hline Pseudomonas & Sporolactoba- & Proteus & & & & & & & \\
\hline Wautersiella & & Pseudomonas & & & & & & & \\
\hline & & Wautersiella & & & & & & & \\
\hline
\end{tabular}




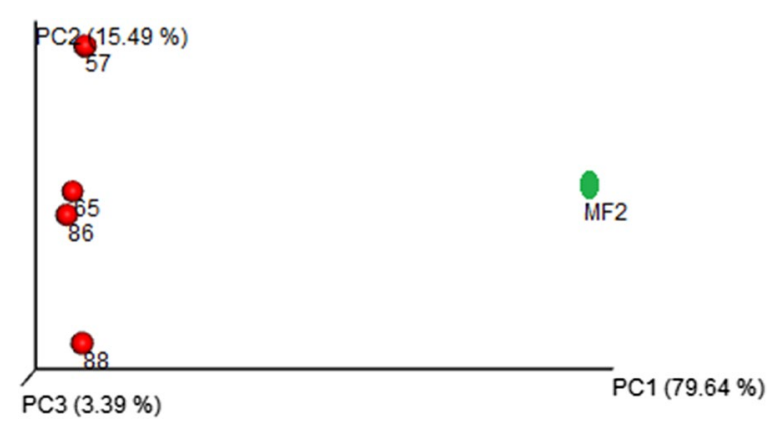

Fig. 7 Comparison of bacterial community of sa-um and Italian salami. All the representatives of Italian salami (Red) are far apart from the sa-um sample (green) in PCoA plot generated using the unweighted Unifac distance $(57,65,86,88$ denotes fasta file SRR5298557, SRR5298558, SRR5298588, SRR5298586, respectively)

gene information (Langille et al. 2013). The imputed metagenomic analysis of the sa-um metagenome identifies antibiotic resistance genes and pro-inflammatory molecules mainly arising from the bacterial families such as Bacillaceae, Bacteroidaceae, Clostridiaceae, Corynebacteriaceae and Enterobacteriaceae indicative of the presence of human pathogenic species in sa-um (Tyx et al. 2016). Resistant gene might have occurred within the gut microbiota of pig and thus serve as a potential route of antimicrobial resistance transmission from animal to human microbiota (Fraqueza 2015). Presence of these resistant genes could be a major concern for this geographic region.

The introduction of NGS technology significantly improved the study of bacterial community present in autochthonous fermented food and the metabolic activity of these microbiota is the key criterion for its unique characteristic sensory attributes. Earlier studies on Italian salami identified the presence of 32 different Staphylococcus and 33 Lactobacillus species from different producers (Połka et al. 2014). Comparison analysis for sa-um with Italian salami revealed that most of the bacterial orders in Italian salami belonged to Lactobacillus, whereas in sa$u m$ it was only $0.3 \%$. This difference may be due to the variation in the carbohydrate or fat content of raw materials used for fermentation in sa-um and Italian salami. This is the first report of characterization of $s a-u m$ a traditional fermented pork fat food. In the present study, high fat content and large number pathogens in sa-um were observed which may cause adverse health risks. This scientific work on an autochthonous fermented pork fat will be useful to the consumers by providing essential and important information on the benefits as well as potential health risks associated with the traditional foodstuff.

\section{Additional file}

Additional file 1: Figure S1. HP-TLC (CAMAG Linomat 5) Chromatogram of sa-um extract $(10 \mu \mathrm{l})$ with Scanning wavelength of $280 \mathrm{~nm}$. Figure S2. Distribution of microbial composition between sa-um and Italian Salami. Studies compared at A: Phylum level and B: Order level.

Abbreviation

ESI+-MS: electrospray ionization-mass spectrometry.

\section{Authors' contributions}

SDM and NSK designed the study. KT and SSS did the sampling. SDM, VK and NSK did all pre-sequencing work for the Illumina and data analysis. RBM analyzed the $\mathrm{ESI}^{+}-\mathrm{MS}$ data. SDM, VK, KT and RBM wrote the manuscript. All authors read and approved the final manuscript.

\section{Author details}

${ }^{1}$ Department of Biotechnology, Mizoram University, Aizawl, Mizoram 796004, India. ${ }^{2}$ Department of Chemistry, Mizoram University, Aizawl, Mizoram 796004 , India. ${ }^{3}$ Dept of Pharmacy, Regional Institute of Paramedical and Nursing Sciences, Aizawl, Mizoram 796017, India. ${ }^{4}$ Biotech Consortium India Ltd, Anuvrat Bhawan, Deen Dayal Upadhyaya Marg, New Delhi 110002, India.

\section{Acknowledgements}

The authors thank Bioinformatics Infrastructure Facility (BT/BI/12/060/2012 (NERBIFMUA) and Advanced Level State Biotech Hub (BT/04/NE/2009 dt. 29.08.2014), Mizoram University, Aizawl sponsored by the Department of Biotechnology (DBT), New Delhi, Govt. of India for providing the facilities for wet-lab and Bioinformatics work.

\section{Competing interests}

The authors declare that they have no competing interests.

Availability of data and materials

Paired end Illumina sequence data from this study were submitted to the NCBI under the BioProject Accession Number SUB2896895.

\section{Consent for publication \\ Not applicable.}

Ethics approval and consent to participate

Not applicable.

\section{Funding}

This research was sponsored by Department of Biotechnology, Govt. of India, New Delhi.

\section{Publisher's Note}

Springer Nature remains neutral with regard to jurisdictional claims in published maps and institutional affiliations.

Received: 25 May 2018 Accepted: 3 October 2018

Published online: 08 October 2018

\section{References}

Akhtar S, Paredes-Sabja D, Torres JA, Sarker MR (2009) Strategy to inactivate Clostridium perfringens spores in meat products. Food Microbiol 26(3):272-277

AOAC (1995) Official methods of analysis of AOAC international. AOAC, Rockville

Aquilanti L, Santarelli S, Silvestri G, Osimani A, Petruzzelli A, Clementi F (2007) The microbial ecology of a typical Italian salami during its natural fermentation. Int J Food Microbiol 120(1-2):136-145

Beuchat LR (2008) Indigenous fermented foods. Biotechnology 9-12:505-559

Borch E, Kant-Muermans ML, Blixt Y (1996) Bacterial spoilage of meat and cured meat products. Int J Food Microbiol 33(1):103-120 
Bray JR, Curtis JT (1957) An ordination of the upland forest communities of southern Wisconsin. Ecol Monogr 27(4):325-349

Caporaso JG, Bittinger K, Bushman FD, Desantis TZ, Andersen GL, Knight R (2009) PyNAST: a flexible tool for aligning sequences to a template alignment. Bioinformatics 26(2):266-267

Caporaso JG, Kuczynski J, Stombaugh J, Bittinger K, Bushman FD, Costello Ek, Fierer N, Peña AG, Goodrich JK, Gordon Jl, Huttley GA, Kelley ST, Knights D, Koenig JE, Ley RE, Lozupone CA, McDonald D, Muegge BD, Pirrung M, Reeder J, Sevinsky JR, Turnbaugh PJ, Walters WA, Widmann J, Yatsunenko T, Zaneveld J, Knight R (2010) QIIME allows analysis of high-throughput community sequencing data. Nat Methods 7(5):335-336

De Mandal S, Zothansanga Panda AK, Bisht SS, Senthil Kumar N (2016) MiSeq HV4 16S rRNA gene analysis of bacterial community composition among the cave sediments of Indo-Burma biodiversity hotspot. Environ Sci Pollut Res 23(12):12216-12226

De Mandal S, Chatterjee R, Senthil Kumar N (2017) Dominant bacterial phyla in caves and their predicted functional roles in $\mathrm{C}$ and $\mathrm{N}$ cycle. BMC Microbiol 17(1):90

DeSantis TZ, Hugenholtz P, Larsen N, Rojas M, Brodie EL, Keller K, Huber T, Dalevi D, Hu P, Andersen GL (2006) Greengenes, a chimera-checked $16 \mathrm{~S}$ rRNA gene database and workbench compatible with ARB. Appl Environ Microbiol 72(7):5069-5072

Edgar RC (2010) Search and clustering orders of magnitude faster than BLAST. Bioinformatics 26(19):2460-2461

Edgar RC, Haas BJ, Clemente JC, Quince C, Knight R (2011) UCHIME improves sensitivity and speed of chimera detection. Bioinformatics 27(16):2194-2200

EFSA and ECDC (2013) The European Union Summary Report on trends and sources of zoonoses, zoonotic agents and food-borne outbreaks in 2011. EFSA J 11(4):1-250

Fraqueza MJ (2015) Antibiotic resistance of lactic acid bacteria isolated from dry-fermented sausages. Int J Food Microbiol 212:76-88

Ghatak S, Yadav RP, Lalrohlui F, Chakraborty P, Ghosh S, Ghosh S, Das M, Pautu $J$, Zohmingthanga J, Senthil Kumar N (2016) Xenobiotic pathway gene polymorphisms associated with gastric cancer in high risk mizo-mongoloid population, Northeast India. Helicobacter 21(6):523-535

Golden NJ, Crouch EA, Latimer H, Kadry AR, Kause J (2009) Risk assessment for Clostridium perfringens in ready-to-eat and partially cooked meat and poultry products. J Food Prot 72(7):1376-1384

Goldman E, Green LH (2015) Practical handbook of microbiology. CRC Press, Boca Raton

Goodacre R, Vaidyanathan S, Bianchi G, Kell DB (2002) Metabolic profiling using direct infusion electrospray ionisation mass spectrometry for the characterisation of olive oils. Analyst 127(11):1457-1462

Hammer BW (1915) Bacteriological studies on the coagulation of evaporated milk. Res Bull 19:117-132

Hartmann R, Meisel H (2007) Food-derived peptides with biological activity: from research to food applications. Curr Opin Biotechnol 18(2):163-169

Hooper L, Summerbell CD, Higgins JPT, Thompson RL, Capps NE, Smith GD, Riemersma RA, Ebrahim S (2001) Dietary fat intake and prevention of cardiovascular disease: systematic review. BMJ 322(7289):757-763

Huang Y, Yi Z, Jin Y, Zhao Y, He K, Liu D, Zhao D, He H, Luo H, Zhang W, Fang Y (2017) New microbial resource: microbial diversity, function and dynamics in Chinese liquor starter. Sci Rep 7(1):14577

Indrasti D, Che Man YB, Mustafa S, Hashim DM (2010) Lard detection based on fatty acids profile using comprehensive gas chromatography hyphenated with time-of-flight mass spectrometry. Food Chem 122:1273-1277

Kurata S, Yamaguchi K, Nagai M (2005) Rapid discrimination of fatty acid composition in fats and oils by electrospray ionization mass spectrometry. Anal Sci 21:1457-1465
Langille MGI, Zaneveld J, Caporaso JG, McDonald D, Knights D, Reyes JA (2013) Predictive functional profiling of microbial communities using 165 rRNA marker gene sequences. Nat Biotechnol 31(9):814-821

Linton M, Connolly M, Houston L, Patterson MF (2014) The control of Clostridium botulinum during extended storage of pressure-treated, cooked chicken. Food Control 37(1):104-108

Lorenzo JM, Franco D (2012) Fat effect on physico-chemical, microbial and textural changes through the manufactured of dry-cured foal sausage Lipolysis, proteolysis and sensory properties. Meat Sci 92(4):704-714

Mayo B, Rachid C, Alegria A, Leite A, Peixoto R, Delgado S (2014) Impact of next generation sequencing techniques in food microbiology. Curr Genomics 15(4):293-309

Nam YD, Seo MJ, Lim SI, Park SL (2012) Genome sequence of Kocuria atrinae C3-8, isolated from jeotgal, a traditional Korean fermented seafood. J Bacteriol 194(21):5996

Nieminen TT, Vihavainen E, Paloranta A, Lehto J, Paulin L, Auvinen P, Solismaa M, Björkroth KJ (2011) Characterization of psychrotrophic bacterial communities in modified atmosphere-packed meat with terminal restriction fragment length polymorphism. Int J Food Microbiol 144(3):336-360

Pennacchia C, Ercolini D, Villani F (2011) Spoilage-related microbiota associated with chilled beef stored in air or vacuum pack. Food Microbiol 28(1):84-93

Połka J, Rebecchi A, Pisacane V, Morelli L, Puglisi E (2014) Bacterial diversity in typical Italian salami at different ripening stages as revealed by high-throughput sequencing of 165 rRNA amplicons. Food Microbiol 46:342-356

Sanders ME, Morelli L, Tompkins TA (2003) Sporeformers as human probiotics: Bacillus, Sporolactobacillus, and Brevibacillus. Compr Rev Food Sci Food Saf 2(3):101-110

Schieber A (2008) Introduction to food authentication in modern techniques for food authentication. In: Sun DW (ed) modern techniques for food authentication. Academic Press, New York

Sekar S, Mariappan S (2007) Usage of traditional fermented products by Indian rural folks and IPR. Indian J Tradit Knowl 6:111-120

Seki H, Shiohara M, Matsumura T, Miyagawa N, Tanaka M, Komiyama A, Kurata $S$ (2003) Prevention of antibiotic-associated diarrhea in children by Clostridium butyricum MIYAIRI. Pediatr Int 45(1):86-90

Shimbo I, Yamaguchi T, Odaka T, Nakajima K, Koide A, Koyama H, Saisho H (2005) Effect of Clostridium butyricum on fecal flora in Helicobacter pylori eradication therapy. World J Gastroenterol 11(47):7520-7524

Sohliya I, Joshi SR, Bhagobaty RK, Kumar R (2009) Tungrymbai-a traditional fermented soybean food of the ethnic tribes of Meghalaya. Indian J Tradit Knowl 8(4):559-561

Solieri L, Dakal TC, Giudici P (2013) Next-generation sequencing and its potential impact on food microbial genomics. Ann Microbiol 63(1):21-37

Tamang JP (1998) Role of microorganisms in traditional fermented foods. Ind Food Ind 17:162-167

Tamang JP, Tamang B, Schillinger U, Guigas C, Holzapfel WH (2009) Functional properties of lactic acid bacteria isolated from ethnic fermented vegetables of the Himalayas. Int J Food Microbiol 135(1):28-33

Tyx RE, Stanfill SB, Keong LM, Rivera AJ, Satten GA, Watson CH (2016) Characterization of bacterial communities in selected smokeless tobacco products using 16S rDNA analysis. PLOS ONE 11(1):e0146939

Yamaoka Y (2008) Helicobacter pylori: molecular genetics and cellular biology. Horizon Scientific Press, Poole 